\title{
Avaliação clínica radiológica da artrodese lombar transforaminal aberta versus minimamente invasiva
}

\author{
Clinical and radiological evaluation of open transforaminal lumbar \\ interbody fusion versus minimally invasive
} Evaluación clínica radiológica de la artrodesis lumbar transforaminal abierta versus mínimamente invasiva

\author{
Cristiano Magalhães Menezes' \\ Roberto Sakamoto Falcon ${ }^{2}$ \\ Marcos Antônio Ferreira Júnior ${ }^{3}$ \\ Johmeson Alencar ${ }^{4}$
}

\section{RESUMO}

Objetivo: a proposta deste trabalho é comparar os resultados clínicos e radiológicos dos pacientes submetidos à artrodese transforaminal aberta e minimamente invasiva. Métodos: quarenta e cinco pacientes foram submetidos à artrodese lombar transforaminal pelo Grupo de Cirurgia Espinhal do Hospital Lifecenter/Ortopédico de Belo Horizonte, no período de Dezembro de 2005 a Maio de 2007, sendo 15 no grupo de artrodese aberta e 30 pacientes do grupo de artrodese minimamente invasiva (MIS). As indicações para a artrodese intersomática foram: doença degenerativa do disco, associada ou não a hérnia de disco ou estenose do canal; espondilolistese de baixo grau espondilolítica ou degenerativa; e síndrome pós-laminectomia/discectomia. As variáveis analisadas foram: tempo de cirurgia, tempo de internação hospitalar, necessidade de hemotransfusão, escala analógica visual de dor (VAS) lombar e dos membros inferiores, Oswestry, índice de consolidação da artrodese e retorno ao trabalho. Resultados: o seguimento mínimo foi de 24

\section{ABSTRACT}

Objective: the aim of this article is to compare the clinical and radiological results of patients who underwent open and minimally invasive transforaminal lumbar interbody fusion. Methods: forty-five patients underwent transforaminal lumbar interbody fusion by the Department of Spine Surgery of Hospital Lifecenter/Ortopédico, between December 2005 and May 2007, 15 of them were in the open interbody fusion group, and 30 in the minimally invasive one. The indications were: the degenerative disc disease associated or not with discal herniation or stenosis; low grade lythic or degenerative spondylolisthesis and postdiscectomy/laminectomy syndrome. The variables analyzed were operative time, length of hospital stay, need for blood transfusion, visual analogical pain scale (VAS), Oswestry functional disability index, solid fusion and return to work. Results: the minimum follow-up was two years. There were eight men and

\section{RESUMEN}

Objetivo: la propuesta de este trabajo es comparar los resultados clínicos y radiológicos de los pacientes sometidos a la artrodesis transforaminal abierta y mínimamente invasiva. Métodos: cuarenta y cinco pacientes fueron sometidos a la artrodesis lumbar transforaminal por el Grupo de Cirugía Espinal del Hospital Lifecenter/Ortopédico de Belo Horizonte, en el periodo de Diciembre de 2005 a Mayo de 2007, siendo 15 en el grupo de artrodesis abierta y 30 pacientes del grupo de artrodesis minimamente invasiva (MIS). Las indicaciones para la artrodesis intersomática fueron: enfermedad degenerativa de disco, asociada o no a hernia de disco o estenosis del canal; espondilolistesis de bajo grado espondilolítica o degenerativa; y síndrome post-laminectomíal discectomía. Las variables analizadas fueron: tiempo de cirugía, tiempo de internación hospitalar, necesidad de hemotransfusión, escala analógica visual de dolor (VAS) lumbar y de los miembros inferiores, Oswestry, indice de consolidación de la artrodesis

\footnotetext{
Trabalho realizado no Hospital Ortopédico/Lifecenter - Belo Horizonte (MG), Brasil.

'Cirurgião do Serviço de Cirurgia Espinhal do Hospital Ortopédico/Lifecenter - Belo Horizonte (MG), Brasil. ${ }^{2}$ Chefe do Serviço de Cirurgia Espinhal do Hospital Ortopédico/Lifecenter - Belo Horizonte (MG), Brasil.

${ }^{3}$ Cirurgião do Serviço de Cirurgia Espinhal do Hospital Oropédico/Lifecenter - Belo Horizonte (MG), Brasil.

${ }^{4}$ Residente do Quarto Ano do Serviço de Cirurgia Espinhal do Hospital Ortopédico/Lifecenter - Belo Horizonte (MG), Brasil. 
meses. Havia oito homens e sete mulheres no Grupo Aberto e 17 homens e 13 mulheres no Grupo MIS. O tempo cirúrgico médio foi de 222 minutos e 221 minutos, respectivamente. Houve melhora significativa da VAS e Oswestry no pós-operatório em ambos os grupos. O tempo de internação hospitalar variou de 3,3 dias para o Grupo Aberto e 1,8 dias para o Grupo MIS. O índice de fusão obtido foi de $93,3 \%$ em ambos os grupos. Houve necessidade de hemotransfusão em três pacientes no Grupo Aberto (20\%) e nenhum caso MIS. Conclusões: a transforaminal lumbar interbody fusion (TLIF) minimamente invasiva apresenta resultados similares em longo prazo quando comparado à TLIF aberta, com os benefícios adicionais de menor morbidade pós-operatória, menor período de internação e reabilitação precoce. seven women in the Open Group, and 17 men and 13 women in the MIS Group. The mean operative time was 222 minutes and 221 minutes, respectively. There was improvement on the VAS and Oswestry scores in both groups. The mean hospital stay was 3.3 days for Open, and 1.8 days for the MIS group. The fusion rate obtained was $93.3 \%$ in both groups. Blood transfusion was necessary for three patients (20\%) of the Open Group. Conclusions: the MIS TLIF has similar results to open TLIF in two years follow-up, with additional benefits of less postoperative morbidity, shorter hospital stay and sooner rehabilitation.

KEYWORDS: Surgical procedures, minimally invasive/ methods; Lumbar vertebrae/ surgery; Lumbar vertebrae/ radiography; Spinal fusion/ methods y regreso al trabajo. Resultados: el seguimiento mínimo fue de 24 meses. Fueron ocho hombres y siete mujeres en el Grupo Abierto y 17 hombres y 13 mujeres en el Grupo MIS. El tiempo quirúrgico promedio fue de $222 \mathrm{mi}$ nutos y 221 minutos, respectivamente. Hubo mejora significativa del VAS y Oswestry en el postoperatorio en ambos grupos. El tiempo de internación hospitalar varió de 3.3 días para el Grupo Abierto y 1.8 días para el Grupo MIS. El índice de fusión obtenido fue de $93.3 \%$ en ambos grupos. Hubo necesidad de hemotransfusión en tres pacientes en el Grupo Abierto (20\%) y ningún caso MIS. Conclusiones: la TLIF mínimamente invasiva presenta resultados similares a largo plazo cuando comparada con TLIF abierta, con los beneficios adicionales de menor morbilidad postoperatoria, menor periodo de internación y rehabilitación precoz.

DESCRIPTORES: Procedimientos quirúrgicos mínimamente invasivos/métodos; Vértebras lumbares/cirugía; Vértebras lumbares/radiografía; Fusión vertebral/métodos

\section{INTRODUÇÃO}

A técnica de artrodese transforaminal (TLIF) é amplamente utilizada por promover altos índices de fusão, restaurar a altura foraminal e manter o alinhamento vertebral. A TLIF aberta vem sendo realizada por vários anos com bons resultados ${ }^{1,2}$. Comparativamente às outras técnicas de artrodese intersomática, apresenta as vantagens de não necessitar de retração e exposição extensas do saco dural e das raízes nervosas, bem como de evitar as estruturas vasculares anteriores à coluna lombar ${ }^{1-3}$.

Contudo, como todos os acessos posteriores à região lombar, a técnica apresenta o inconveniente de promover extensa exposição cirúrgica necessária à criação do leito de artrodese e visualização dos marcadores anatômicos para a correta introdução dos parafusos pediculares ${ }^{1-16}$. Essa extensa dissecção de partes moles é a responsável por maior dor no pós-operatório, por volumosos sangramentos, pela recuperação mais lenta e pela perda a longo prazo da função espinal.

A técnica de artrodese lombar transforaminal minimamente invasiva (MIS TLIF) tem sido apresentada nos últimos anos como uma opção aos procedimentos tradicionais abertos, oferecendo menores incisões, menor trauma às partes moles e recuperação mais precoce. O objetivo deste estudo é comparar os resultados clínicos da cirurgia convencional aberta e a MIS TLF.

\section{MÉTODOS}

Entre Dezembro de 2005 e Maio de 2007, 45 pacientes submeteram-se a uma TLIF, sendo que 15 pertenciam a um grupo de cirurgia convencional aberta e 30 ao grupo de MIS TLIF. As indicações para a cirurgia foram: doença degenerativa do disco associada ou não à hérnia discal ou à estenose do canal lombar, espondilolistese de baixo grau espondilolítica ou degenerativa e síndrome pós-laminectomia/discectomia. Todos os pacientes tinham exames pré-operatórios que incluíam radiografias estáticas e dinâmicas da coluna lombar e ressonância magnética, tendo falhado o tratamento conservador por um mínimo de seis meses antes da cirurgia. O seguimento pós-operatório mínimo foi de 24 meses (variando de 24 a 42 meses). 
Os dados demográficos e cirúrgicos dos 45 pacientes foram coletados e analisados. Dentre os 30 pacientes do Grupo MIS, estavam incluídos os primeiros casos da curva de aprendizado.

As variáveis analisadas foram: tempo de cirurgia, tempo de internação, escala visual de dor (VAS) lombar e de membros inferiores (MMII) pré e pós-operatórios, escala funcional de Oswestry pré e pós-operatória, necessidade de hemotransfusão, complicações, retorno ao trabalho e fusão radiológica.

Foram realizadas avaliações no pré-operatório, pósoperatório imediato, seis semanas, três meses, seis meses e anualmente após essa última.

A análise estatística foi realizada com o programa SPSS, versão 10.0. Utilizou-se para tais análises o teste $t$ de Student e a análise de variância, considerando como significância o valor de $\mathrm{p}<0,05$.

\section{Técnica para a MIS TLIF}

O paciente é posicionado em decúbito ventral, sob anestesia geral, em um suporte de coluna e uma mesa cirúrgica radiotransparente. Uma incisão de $2,5 \mathrm{~cm}$ de comprimento é realizada a uma distância de $4,5 \mathrm{~cm}$ da linha média. Dilatadores sequenciais são usados até a colocação de um tubo de $22 \mathrm{~mm}$ de diâmetro e profundidade apropriada para cada paciente, sobre o complexo facetário. A facetectomia total é realizada com uma broca de alta rotação para a exposição do aspecto póstero-lateral do disco intervertebral. A margem lateral do ligamento amarelo é ressecada para a exposição das raízes descendente e transeunte. Caso seja necessária uma descompressão mais ampla do canal central e das estruturas contralaterais, isso poderá ser obtido inclinando-se o tubo medialmente e realizando uma descompressão sublaminar monoportal bilateral.

A completa discectomia e a correta preparação das plataformas vertebrais são realizadas com o auxílio de curetas, raspas e dilatadores. O cuidado para a criação de um bom leito para a artrodese é fundamental e inerente à técnica escolhida pelo cirurgião. Enxerto autólogo de crista ilíaca é obtido percutaneamente pela utilização de uma trefina. $\mathrm{O}$ enxerto é colocado em quantidade adequada no espaço intersomático e dentro do espaçador. Foi utilizado um espaçador de PEEK autodistrator. A artrodese posterior do lado contralateral é realizada, o retrator tubular é retirado cuidadosamente, e qualquer sangramento da musculatura paravertebral pode ser controlado com o uso do cautério bipolar.

A construção com parafusos pediculares percutâneos é, então, iniciada, utilizando-se uma agulha de Jamshidi para ter acesso ao pedículo, por meio de um trajeto de lateral para medial. É realizada a introdução de um fio guia, seguida de uma série de dilatadores tubulares. O tap é utilizado até a profundidade desejada do parafuso a ser escolhido. O parafuso multiaxial permite ao sistema a mensuração da haste lordótica a ser utilizada, esta é introduzida por um arco através de uma incisão proximal puntiforme. Todo o sistema é submetido à compressão para a estabilidade necessária. As feridas são suturadas com pontos intradérmicos.

\section{Técnica para a TLIF aberta}

Uma incisão na linha média posterior é realizada. A fáscia toracolombar é exposta e uma dissecção subperióstea desinsere a musculatura paravertebral dos processos espinhosos e das lâminas. Construções bilaterais com parafusos pediculares e hastes são realizadas, bem como uma facetectomia com preservação laminar e do processo espinhoso. Segue-se, então, a discectomia, preparação do espaço intersomático, enxertia óssea autóloga e inserção do espaçador intersomático, e a artrodese posterior e pósterolateral. A ferida é copiosamente irrigada e as suturas são realizadas por planos.

\section{RESULTADOS}

Os dados demográficos eram comparáveis entre os grupos. A média da idade do Grupo Aberto era de 45,9 anos (variando entre 27 e 65 anos) e do Grupo MIS, 45,2 anos (variando entre 18 e 71 anos). Havia oito homens e sete mulheres no Grupo Aberto e 17 homens e 13 mulheres no Grupo MIS. No Grupo Aberto, 11 casos foram operados em um nível e quatro em dois, enquanto no Grupo MIS 27 foram operados em um nível e três em dois, sempre entre L3 e S1. O tempo cirúrgico médio foi de 222 e 221 minutos, respectivamente. Houve necessidade de hemotransfusão em três pacientes no Grupo Aberto (20\%) e nenhum caso no Grupo MIS $(\mathrm{p}<0,05)$. O tempo de internação hospitalar variou de 3,3 dias para o Grupo Aberto e 1,8 dias para o Grupo MIS $(\mathrm{p}<0,05)$.

Houve melhora significativa da VAS e Oswestry no pós-operatório em ambos os grupos. A VAS lombar do pré-operatório decresceu de 8,2 para 2,3 no Grupo Aberto (melhora de $72 \%$ ), $\mathrm{p}<0,05$, e de 8,4 para $1,7(80 \%)$ no Grupo MIS $(p<0,05)$, com dois anos de seguimento. Essa diferença não foi estatisticamente significante $(\mathrm{p}=0,11)$, demonstrando apenas uma tendência a uma VAS menor no Grupo MIS. A VAS dos MMII de 7,8 decresceu para 0,9 $(88,5 \%)$ no Grupo Aberto $(\mathrm{p}=0.0002)$, e de 7,5 para 0,9 $(88 \%)$ no Grupo MIS ( $\mathrm{p}=0,0004)$. A escala de Oswestry pré-operatória diminuiu de 42,3 para $13,5 \%$ no Grupo Aberto $(\mathrm{p}<0,05)$, e de 46,6 para $12,6 \%$ no Grupo MIS $(p<0,05)$, resultados totalmente comparáveis.

O tempo médio de retorno ao trabalho foi de 2,7 meses para o Grupo Aberto e de 45 dias para o Grupo MIS $(\mathrm{p}<0,05)$. Dois pacientes não retornaram ao trabalho no Grupo Aberto. Todos os pacientes do Grupo MIS retornaram ao trabalho.

O índice de fusão obtido foi de $93,3 \%$ em ambos os grupos (uma pseudoartrose no Grupo Aberto e duas no Grupo MIS), verificado por meio de tomografia computadorizada com cortes finos pela presença de trabéculas ósseas entre os corpos vertebrais (Figura 1).

Foram obtidas complicações como, por exemplo, duas reoperações no Grupo Aberto, uma por um parafuso posicionado lateralmente e uma para correção de uma fístula 
liquórica. Ainda neste grupo, houve uma soltura de haste unilateral e uma subsidência do espaçador intersomático, ambas as situações sem maiores repercussões. No Grupo MIS, houve a necessidade de converter, no início da curva de aprendizado, parcialmente para cirurgia aberta em dois pacientes, um hematoma perirradicular com radiculite inflamatória pós-operatória em um paciente, uma paresia temporária de L5 em um paciente e uma pequena durotomia acidental tratada com cola de fibrina.

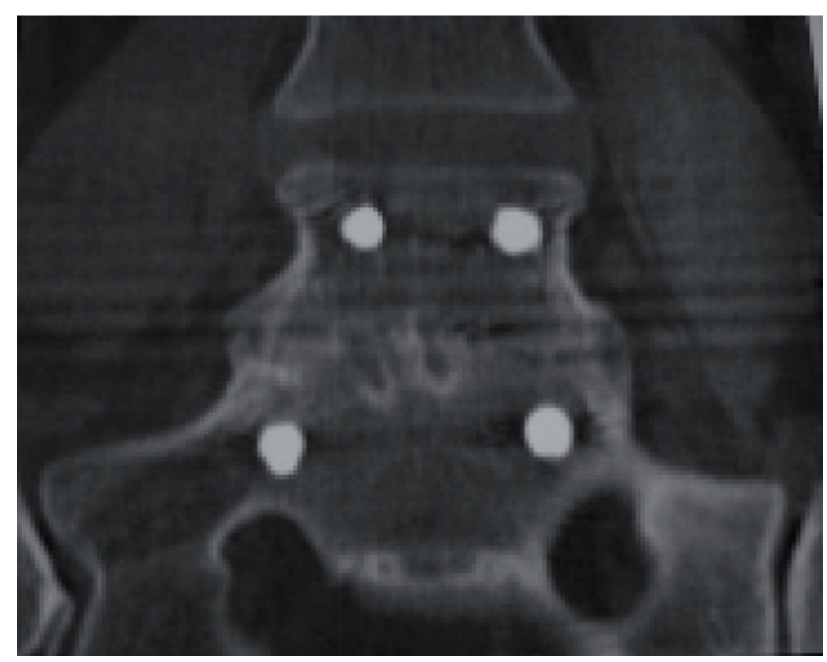

Figura 1

Cortes tomográficos evidenciando continuidade das trabéculas ósseas entre os corpos vertebrais.

\section{DISCUSSÃO}

A cirurgia de artrodese lombar associa-se a uma extensa dissecção das partes moles e vários autores já relataram as consequências negativas deste procedimento, com aumento significativo da morbidade. A MIS TLIF, por se tratar de um acesso parassagital entre o multifidus e o longissimus (eretor espinal), preserva a banda de tensão posterior natural criada pelos ligamentos inter e supraespinais, bem como a inserção, vascularização e inervação da musculatura paravertebral ${ }^{17-20}$.

$\mathrm{O}$ acesso transforaminal, aberto ou minimamente invasivo, promove uma completa exposição do aspecto póstero-lateral do disco intervertebral, necessitando de uma mínima retração das raízes nervosas e do saco dural para a realização da artrodese intersomática e implantação do espaçador intersomático. Os retratores são utilizados meramente como protetores durante a preparação adequada do espaço intersomático e colocação do enxerto ósseo e do espaçador. O afastamento é, portanto, unilateral e mínimo, reduzindo significativamente o risco de lesão neural por tração e manipulação. Os parafusos percutâneos também asseguram que as partes moles dos níveis adjacentes ao operado permaneçam intactas, evitando o surgimento de uma instabilidade iatrogênica proximal $^{20-22}$. (Figuras 2, 3 e 4).

No presente estudo, outra vantagem clara da MIS TLIF é a redução do sangramento intraoperatório, quando

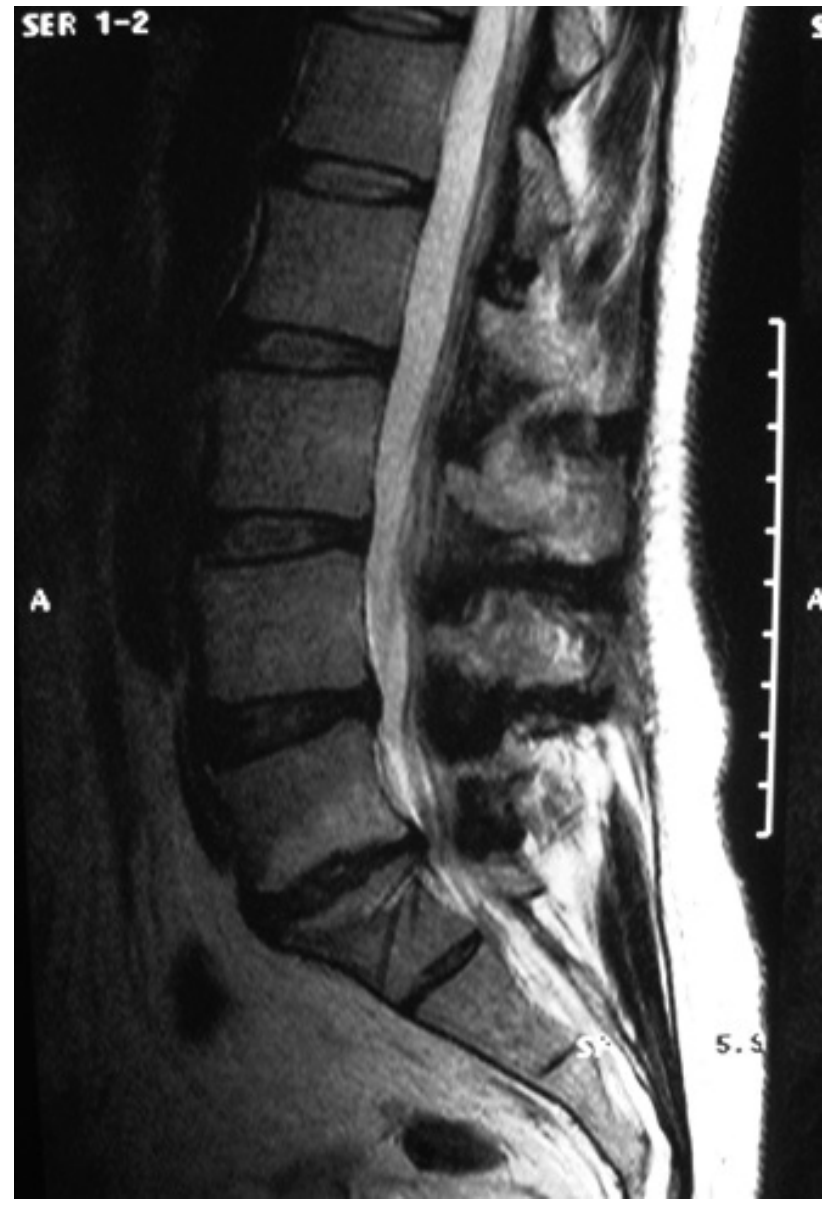

Figura 2

Ressonância magnética pré-operatória, demonstrando discopatia degenerativa L5S1 associada à estenose do canal lombar.
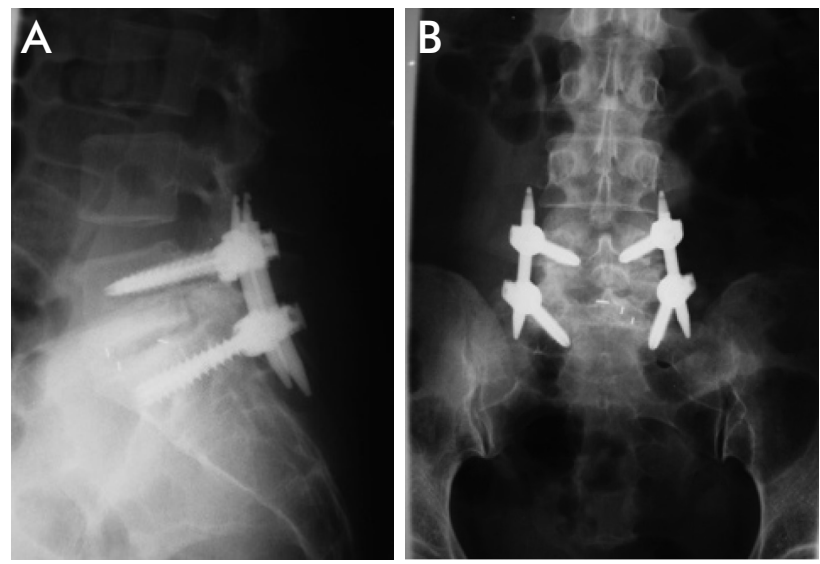

Figura $3 A$ e $3 B$

Radiografias em AP e perfil de paciente submetido a MIS TLIF L5ST.

comparado à TLIF aberta. Perda sanguínea substancial é frequentemente relatada na literatura quando a artrodese aberta é realizada. Essa redução do sangramento observada nos casos MIS, praticamente exclui a necessidade de hemotransfusão e de seus riscos associados.

Ambos os grupos obtiveram uma melhora dos índices de VAS lombar e dos MMII, bem como da escala de 


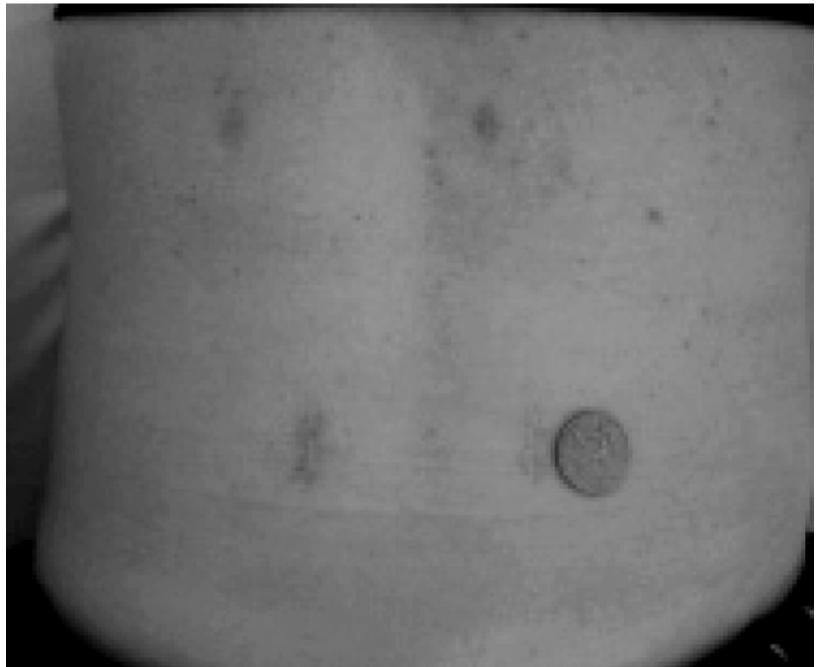

Figura 4

Cicatrizes pós-operatórias.

Oswestry (Gráficos 1, 2 e 3). Esperava-se que os grupos mantivessem esta relação de equivalência, demonstrando os benefícios de uma artrodese transforaminal, seja por via aberta ou minimamente invasiva. Porém, como já descrito por vários autores, os benefícios imediatos de uma redução significativa da morbidade cirúrgica justificam a utilização da técnica minimamente invasiva, que se demonstrou tão eficaz e segura quanto à cirurgia aberta. $\mathrm{Khoo}^{23}$, no World Congress of Minimally Invasive Spine Surgery and Techniques, de 2008, demonstrou resultados semelhantes aos do presente estudo com dois anos de segmento. Porém o autor também observou que, com quatro anos de segmento os pacientes do Grupo MIS tendiam a permanecer com os seus escores baixos, enquanto o Grupo Aberto seria propenso a um aumento da VAS lombar e da Oswestry.

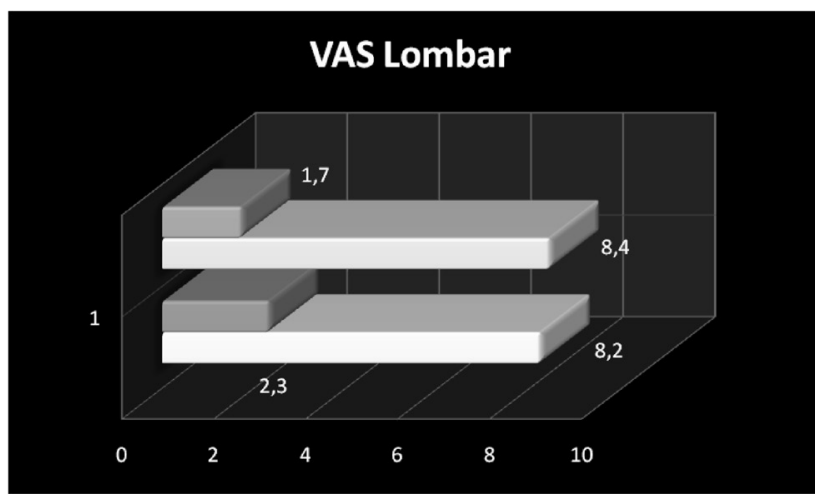

Gráfico 1

Avaliação da VAS lombar no pré e pós-operatório.

O tempo médio de retorno ao trabalho foi de 2,7 meses para o Grupo Aberto, sendo que dois pacientes não retornaram ao trabalho. Ambos já estavam afastados de sua atividade laboral por um período superior a seis

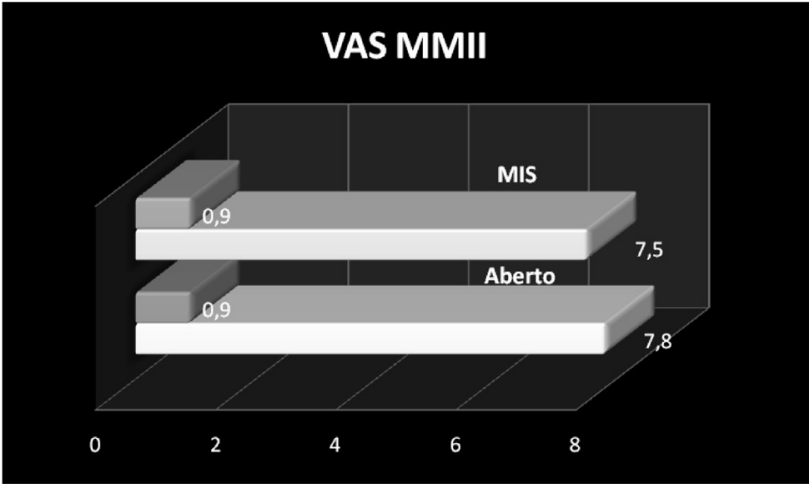

Gráfico 2

Avaliação da VAS dos MMIl no pré e pós-operatório.

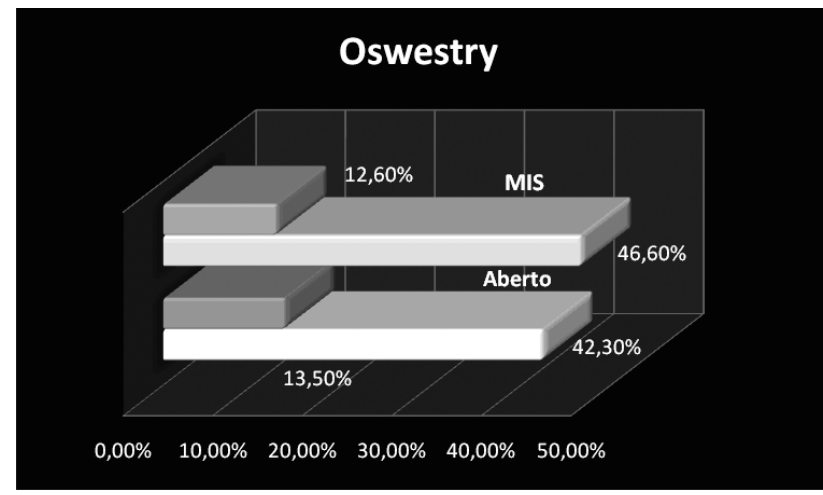

Gráfico 3

Avaliação da escala funcional de Oswestry no pré e pósoperatório.

meses antes da cirurgia. O tempo médio de retorno ao trabalho no Grupo MIS foi de 45 dias $(\mathrm{p}<0,05)$, sendo que dos 30 pacientes operados, 26 trabalhavam e retornaram às suas funções.

Uma curva de aprendizado mais longa e um treinamento adequado são fundamentais para o desenvolvimento seguro desta técnica. Diferentemente dos acessos tradicionais abertos, as cirurgias minimamente invasivas estão limitadas à área de interesse cirúrgico e alguns pontos anatômicos de interesse, em um campo de visão limitado $^{14}$. A familiaridade com o procedimento permite que o cirurgião possa realizar, de maneira segura, a cirurgia, sem a necessidade da exposição de estruturas anatômicas que não serão tratadas cirurgicamente.

\section{CONCLUSÃO}

Os resultados com dois anos de pós-operatório demonstram que a artrodese transforaminal aberta e a minimamente invasiva apresentam bons resultados clínicos e radiológicos. A técnica minimamente invasiva é uma opção efetiva e segura, levando a uma menor morbidade cirúrgica, diminuindo o sangramento, o tempo de internação e permitindo um retorno precoce ao trabalho. 


\section{REFERÊNCIAS}

1. Harms J, Rolinger H. [A one-stager procedure in operative treatment of spondylolisthesis: dorsal tractionreposition and anterior fusion (author's transl)]. Z Orthop Ihre Grenzgeb. 1982;120(3):343-7. German.

2. Menezes CM, Falcon RS, Ferreira Júnior MA, Oliveira DA, Freire SG. Experiência inicial com a técnica de artrodese lombar minimamente invasiva por via transforaminal (MIS TLIF). Coluna /Columna. 2007;6(3):141-8.

3. Menezes CM, Ferreira Júnior MA, Falcon RS, Oliveira DA. Artrodese minimamente invasiva para espondilolisteses de baixo grau. Coluna/Columna. 2008;7(3):241-5.

4. Zhu XZ, Parnianpour M, Nordin M, Kahanovitz N. Histochemistry and morphology of erector spinae muscle in lumbar disc herniation. Spine (Phila Pa 1976). 1989;14(4):391-7.

5. Kawaguchi Y, Matsui H, Gejo R, Tsuji H. Preventive measures of back muscle injury after posterior lumbar spine surgery in rats. Spine (Phila $\mathrm{Pa}$ 1976). 1998;23(21):2282-7; discussion 2288.

6. Weber BR, Grob D, Dvorák J, Müntener M. Posterior surgical approach to the lumbar spine and its effect on the multifidus muscle. Spine (Phila Pa 1976). 1997;22(15):1765-72.

7. Kawaguchi Y, Yabuki S, Styf J, Olmarker K, Rydevik B, Matsui H, et al. Back muscle injury after posterior lumbar spine surgery. Topographic evaluation of intramuscular pressure and blood flow in the porcine back muscle during surgery. Spine (Phila $\mathrm{Pa}$ 1976). 1996;21(22):2683-8.

8. Naylor A. Late results of laminectomy for lumbar disc prolapse. A review after ten to twenty-five years. J Bone Joint Surg Br. 1974;56(1):17-29.

9. Jackson RK. The long-term effects of wide laminectomy for lumbar disc excision. A review of 130 patients. J Bone Joint Surg Br. 1971;53(4): 609-16.
10.Gejo R, Matsui H, Kawaguchi Y, Ishihara H, Tsuji H. Serial changes in trunk muscle performance after posterior lumbar surgery. Spine (Phila Pa 1976). 1999;24(10):1023-8.

11.Kawaguchi Y, Matsui H, Tsuji H. Back muscle injury after posterior lumbar spine surgery. A histologic and enzymatic analysis. Spine (Phila $\mathrm{Pa}$ 1976). 1996;21(8):941-4.

12.Kawaguchi Y, Matsui H, Tsuji H. Back muscle injury after posterior lumbar spine surgery. Part 2: Histologic and histochemical analyses in humans. Spine (Phila Pa 1976). 1994;19(22):2598-602.

13.Mayer TG, Vanharanta H, Gatchel RJ, Mooney V, Barnes D, Judge L, et al. Comparison of CT scan muscle measurements and isokinetic trunk strength in postoperative patients. Spine (Phila Pa 1976). 1989;14(1): 33-6.

14.Rantanen J, Hurme M, Falck B, Alaranta H, Nykvist F, Lehto M, et al. The lumbar multifidus muscle five years after surgery for a lumbar intervertebral disc herniation. Spine (Phila Pa 1976). 1993;18(5):568-74.

15.Sihvonen T, Herno A, Paljärvi L, Airaksinen O, Partanen J, Tapaninaho A. Local denervation atrophy of paraspinal muscles in postoperative failed back syndrome. Spine (Phila Pa 1976). 1993;18(5):575-81.

16.Styf JR, Willén J. The effects of external compression by three different retractors on pressure in the erector spine muscles during and after posterior lumbar spine surgery in humans. Spine (Phila Pa 1976). 1998;23(3):354-8.

17.Foley KT, Holly LT, Schwender JD. Minimally invasive lumbar fusion. Spine (Phila Pa 1976). 2003;28(15 Suppl):S26-35. Review.
18.Isaacs RE, Podichetty VK, Santiago P, Sandhu FA, Spears J, Kelly K, et al. Minimally invasive microendoscopyassisted transforaminal lumbar interbody fusion with instrumentation. J Neurosurg Spine. 2005;3(2):98-105.

19. Scheufler KM, Dohmen H, Vougioukas VI. Percutaneous transforaminal lumbar interbody fusion for the treatment of degenerative lumbar instability. Neurosurgery. 2007;60(4 Suppl 2):203-12; discussion 212-3.

20.Harms JG, Jeszenszky D. The unilateral, transforaminal approach for posterior lumbar interbody fusion. Orthop Traumatol. 1998;6(1):88-99.

21.Lowe TG, Tahernia AD, O'Brien MF, Smith DA. Unilateral transforaminal posterior lumbar interbody fusion (TLIF): indications, technique, and 2 -year results. J Spinal Disord Tech. 2002;15(1):31-8.

22.Rosenberg WS, Mummaneni PV. Transforaminal lumbar interbody fusion: technique, complications, and early results. Neurosurgery. 2001;48(3):569-74; discussion 574-5.

23.Khoo LT. Long-term outcomes of minimally invasive versus open transforaminal lumbar interbody fusion: surgical results and outcomes in a series of 128 patients. In: World Congress of Minimally Invasive Spine Surgery and Techniques (WCMISST), Hawaii; 05 a 07 de June 2008.

\section{Correspondência}

Cristiano Menezes

Hospital Ortopédico de Belo Horizonte

Rua Professor Otávio Coelho de Magalhães, 111 , Mangabeiras

CEP 30210-300 - Belo Horizonte

(MG), Brasil

E-mail: cristiano@vertebral.com.br 